\title{
Bennett's linkage and the cylindroid
}

\author{
Alba Perez *, J.M. McCarthy * \\ Robotics and Automation Laboratory, Department of Mechanical and Aerospace Engineering, \\ University of California, 02708-3975, Irvine, CA 92697, USA
}

Received 20 August 2001; received in revised form 31 May 2002; accepted 2 June 2002

\begin{abstract}
Bennett's linkage is a spatial $4 \mathrm{R}$ closed chain that can move with one degree of freedom. The set of relative displacement screws that form the one-dimensional workspace of this device defines a ruled surface known as a cylindroid. The cylindroid is generally obtained as a result of a real linear combination of two screws. Thus, the workspace of Bennett's linkage is directly related to a one-dimensional linear subspace of screws. In this paper, we examine in detail Bennett's linkage and its associated cylindroid, and introduce a reference pyramid which provides a convenient way to relate the two. These results are fundamental to efficient techniques for solving the synthesis equations for spatial RR chains.

(C) 2002 Elsevier Science Ltd. All rights reserved.
\end{abstract}

Keywords: Bennett linkage; Cylindroid; Spatial linkage; Dual quaternions; Constraint manifold

\section{Introduction}

Bennett's linkage [1] is the $4 \mathrm{R}$ closed chain that guides a coupler through a spatial trajectory. The planar $4 \mathrm{R}$ and spherical $4 \mathrm{R}$ linkages have couplers that move along a plane or on a sphere, respectively. The movement of Bennett's linkage depends upon a specific relation among the physical dimensions of the four links and is termed over-constrained. The general spatial $4 \mathrm{R}$ closed chain forms a statically indeterminate structure.

In this paper, we examine the dual quaternion form of the set of displacements of the coupler of Bennett's linkage, termed its constraint manifold [2]. Each of these displacements defines a screw axis, S, together with a rotation angle $\phi$ and slide $k$ around and along this axis.

\footnotetext{
"Corresponding authors. Tel.: +1-949-824-5406; fax: +1-949-824-8585.

E-mail addresses: maperez@uci.edu (A. Perez),jmmccart@uci.edu (J.M. McCarthy).
} 
Huang [3,4] has shown that this one-dimensional set of axes forms a cylindroid, which is a ruled surface originally studied by Plücker ([5-7]).

Our goal is to formulate the equation of this cylindroid and locate it relative to the Bennett linkage. This is done by defining a rectangular pyramid constructed from the reference position of the linkage, which is then used to locate the principal axes of the cylindroid. This formulation is adapted from an approach initially introduced by $\mathrm{Yu}$ [8], and is fundamental to design techniques for RR chains [9].

\section{Bennett's linkage}

Consider separately the input and output cranks of Bennett's linkage which we denote as GW and $\mathrm{HU}$, respectively (Fig. 1). For the input crank, let $\mathrm{N}_{1}$ be the common normal between the axes $\mathrm{G}$ and $\mathrm{W}$ of the revolute joints, and let $\mathbf{B}$ and $\mathbf{P}$ be their points of intersection with $\mathrm{N}_{1}$. The angle $\alpha$ and distance $a$ from $\mathrm{G}$ to $\mathrm{W}$ measured around and along $\mathrm{N}_{1}$ define the dimensions of the input crank, and can be assembled into the dual number $\hat{\alpha}=(\alpha, a)$. The output crank HU of Bennett's linkage has the same dimensions, that is $\hat{\alpha}=(\alpha, a)$ defines the angle and distance from $\mathrm{H}$ to $\mathrm{U}$ around and along its common normal $\mathrm{N}_{2}$. Let $\mathbf{Q}$ and $\mathbf{C}$ be the intersections of $\mathrm{N}_{2}$ with $\mathrm{H}$ and $\mathrm{U}$, respectively.

In order to form the linkage, we assemble the base link so that the points $\mathbf{B}$ and $\mathbf{Q}$ lie on the common normal between the fixed joint axes $\mathrm{G}$ and $\mathrm{H}$, and $\mathbf{P}$ and $\mathbf{C}$ lie on the common normal to the two moving axes $\mathrm{W}$ and $\mathrm{U}$. The dimensions of these two links are to be the same, such that $\hat{\gamma}=(\gamma, g)$ defines the angle and distance between these axes around the common normal.

It is possible to show that in order to achieve this assembly, the dimensions of Bennett's linkage must satisfy the relation [10],

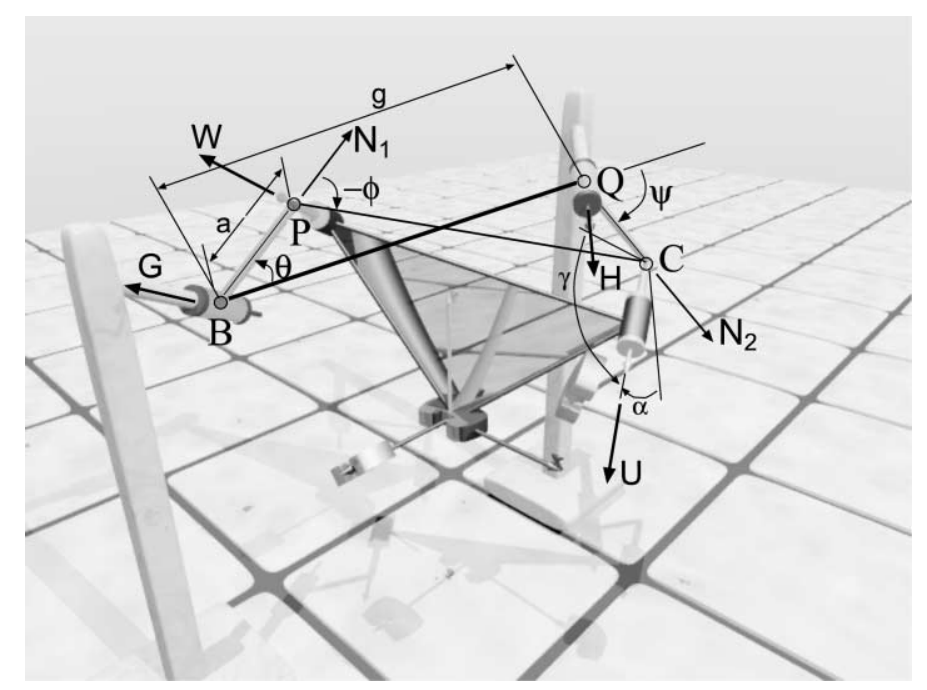

Fig. 1. Bennett's linkage defined by axes GWUH and their common normals. 


$$
\frac{\sin \alpha}{a}=\frac{\sin \gamma}{g}
$$

For the analysis of the Bennett's linkage, we introduce a frame $F$ with origin at the point $\mathbf{B}$, its $z$-axis along the fixed axis $\mathrm{G}$, and its $x$-axis along the common normal between $\mathrm{G}$ and $\mathrm{H}$.

The input-output equation for Bennett's linkage [11] is derived from the fact that that the points $\mathbf{P}$ and $\mathbf{C}$ must remain separated by the distance $g$ as the linkage moves. This constraint equation,

$$
(\mathbf{C}-\mathbf{P}) \cdot(\mathbf{C}-\mathbf{P})=g^{2}
$$

yields

$$
\tan \frac{\psi}{2}=k^{ \pm} \tan \frac{\theta}{2}
$$

where

$$
k^{+}=\frac{\sin \frac{\alpha+\gamma}{2}}{\sin \frac{\alpha-\gamma}{2}}, \quad \text { and } \quad k^{-}=\frac{\cos \frac{\alpha-\gamma}{2}}{\cos \frac{\alpha+\gamma}{2}} .
$$

Hunt [5] shows that $k^{+}$is the correct root for the conventions that we have chosen for the angles $\alpha$ and $\gamma$. The negative root yields the constant associated with a different sign convention. In what follows we focus on the positive root, which we denote at $k$, and drop the + superscript.

To relate the output angle $\psi$ to the coupler angle $\phi$ (the angle measured around W that defines the position of the coupler WU relative to the input crank), we write the loop equations for the linkage. We equate the two expressions of the point $\mathbf{C}$ to obtain

$$
\begin{aligned}
& g(\cos \psi-\cos \phi)=0, \\
& g \sin \alpha(\sin \psi-\sin \phi)=0 .
\end{aligned}
$$

Thus, we see that the coupler angle $\phi$ is equal to the output angle $\psi$. This allows us to use (3) to compute

$$
\tan \frac{\phi}{2}=k \tan \frac{\theta}{2}
$$

where, as we have seen previously, $k$ depends only on the linkage dimensions.

\section{The constraint manifold}

The workspace of a tool attached to the coupler of Bennett's linkage is the set of positions and orientations that it can reach. It is defined by constraining the kinematics equations of the RR chain that forms the input crank

$$
[D(\theta, \phi)]=[G][Z(\theta, 0)][X(\alpha, a)][Z(\phi, 0)][H] .
$$

The transformation $[G]$ locates the standard frame $F$ in a global reference frame, and $[H]$ defines the location of the tool frame relative to the coupler frame. We can select any of the positions in 
the workspace of the tool to be a reference position with input and coupler angles $\theta_{0}$ and $\phi_{0}$. The set of displacements of the tool relative to this reference position are defined by

$$
\left[D_{0 \theta}(\Delta \theta, \Delta \phi)\right]=[D(\theta, \phi)]\left[D\left(\theta_{0}, \phi_{0}\right)\right]^{-1}
$$

where $\Delta \theta=\theta-\theta_{0}$ and $\Delta \phi=\phi-\phi_{0}$.

We can write the relative displacements (8) as a composition of rotations about the joint axes $G$ and $\mathrm{W}$,

$$
\left[D_{0 \theta}(\Delta \theta, \Delta \phi)\right]=[T(\Delta \theta, \mathrm{G})][T(\Delta \phi, \mathrm{W})]
$$

where

$$
\begin{aligned}
& {[T(\Delta \theta, \mathrm{G})]=[G][Z(\theta, 0)]\left[Z\left(\theta_{0}, 0\right)\right]^{-1}[G]^{-1}} \\
& {[T(\Delta \phi, \mathrm{W})]=[G]\left[Z\left(\theta_{0}, 0\right)\right][X(\alpha, a)][Z(\phi, 0)]\left([G]\left[Z\left(\theta_{0}, 0\right)\right][X(\alpha, a)]\left[Z\left(\phi_{0}, 0\right)\right]\right)^{-1}}
\end{aligned}
$$

The two matrices in (9) are displacements defined as a rotation of $\Delta \phi$ about W, followed by a rotation about $\mathrm{G}$ by the angle $\Delta \theta$. Notice that the axis $\mathrm{W}$ is located as defined by the reference crank angle $\theta_{0}$. The composition of these displacements defines a screw axis $\mathrm{S}$, a relative rotation angle $\Delta \psi=\psi-\psi_{0}$ and a relative slide $\Delta t=t-t_{0}$, around and along this axis. This composition is easily computed using dual quaternion multiplication [11].

\subsection{Dual quaternions}

Let $\mathbf{G}$ be the unit vector defining the direction of the axis $G$, and let $\mathbf{p}$ be a point on this line, then $G=(\mathbf{G}, \mathbf{p} \times \mathbf{G})$ is the dual vector that defines it. These six coordinates are the well-known Plücker coordinates of a line. Similarly, we have $\mathbf{W}=(\mathbf{W}, \mathbf{q} \times \mathbf{W})$, where $\mathbf{W}$ is the direction of $\mathbf{W}$ and $\mathbf{q}$ is a point on this line.

If we now denote the pair $\Delta \hat{\psi}=(\psi, t)$, which we call a dual angle, we can then present the result of the composition of the two displacements about $\mathrm{G}$ and $\mathrm{W}$ as

$$
\cos \frac{\Delta \hat{\psi}}{2}=\cos \frac{\Delta \theta}{2} \cos \frac{\Delta \phi}{2}-\sin \frac{\Delta \theta}{2} \sin \frac{\Delta \phi}{2} \mathrm{G} \cdot \mathrm{W}
$$

and

$$
\sin \frac{\Delta \hat{\psi}}{2} \mathrm{~S}=\sin \frac{\Delta \theta}{2} \cos \frac{\Delta \phi}{2} \mathrm{G}+\sin \frac{\Delta \phi}{2} \cos \frac{\Delta \theta}{2} \mathrm{~W}+\sin \frac{\Delta \theta}{2} \sin \frac{\Delta \phi}{2} \mathrm{G} \times \mathrm{W} .
$$

These formulas can be obtained in several ways, the easiest, perhaps, is by dual quaternion multiplication $[2,6,12]$.

Expanding (11) according to the geometry of the Bennett's linkage, we obtain

$$
\cos \frac{\Delta \theta}{2} \cos \frac{\Delta \phi}{2}=\cos \frac{\Delta \psi}{2}-\frac{\frac{\Delta t}{2} \sin \frac{\Delta \psi}{2}}{a \sin \alpha}
$$


Divide this result into (12) to obtain

$$
\mathrm{F}=M(\Delta \psi)\left(1, \frac{\frac{\Delta t}{2}}{\tan \frac{\Delta \psi}{2}}\right) \mathrm{S}=\tan \frac{\Delta \theta}{2} \mathrm{G}+\tan \frac{\Delta \phi}{2} \mathrm{~W}+\tan \frac{\Delta \theta}{2} \tan \frac{\Delta \phi}{2} \mathrm{G} \times \mathrm{W},
$$

where

$$
M(\Delta \psi)=\frac{a \sin \alpha \sin \frac{\Delta \psi}{2}}{a \sin \alpha \cos \frac{\Delta \psi}{2}-\frac{\Delta t}{2} \sin \frac{\Delta \psi}{2}}
$$

is the magnitude of each screw and $\mathrm{S}$ is the Plücker coordinates of its axis. The parameter

$$
P=\frac{\frac{\Delta t}{2}}{\tan \frac{\Delta \psi}{2}}
$$

is called the pitch of the screw.

The axes of the screws $F$ are the axes of relative displacement screws of the coupler of Bennett's linkage, and form the screw surface for the movement.

\subsection{The screw surface}

Let $\theta_{0}$ and $\phi_{0}$ denote the reference configuration for the drive and coupler angles of the Bennett linkage, then the relative displacement screws can be computed using (14), to be

$$
\mathrm{F}\left(\theta, \theta_{0}\right)=\tan \frac{\theta-\theta_{0}}{2} \mathrm{G}+\tan \frac{\phi-\phi_{0}}{2} \mathrm{~W}+\tan \frac{\theta-\theta_{0}}{2} \tan \frac{\phi-\phi_{0}}{2} \mathrm{G} \times \mathrm{W}
$$

We expand this equation using the standard reference frame to define $\mathrm{G}$ and $\mathrm{W}$, and the relationship between $\theta$ and $\phi$ given by (6). Factor the common terms from the components of $F$ to obtain the simplified expression $\mathrm{F}^{\prime}$,

$$
\mathrm{F}^{\prime}\left(\theta, \theta_{0}\right)=\left(\left\{\begin{array}{c}
-(\mathrm{c} \alpha-\mathrm{c} \gamma) \mathrm{s} \alpha \mathrm{s}\left(\frac{\theta+\theta_{0}}{2}\right) \\
(\mathrm{c} \alpha-\mathrm{c} \gamma) \mathrm{s} \alpha \mathrm{c}\left(\frac{\theta+\theta_{0}}{2}\right) \\
\mathrm{s} \alpha\left(\mathrm{c}\left(\frac{\theta-\theta_{0}}{2}\right) \mathrm{s} \alpha-\mathrm{c}\left(\frac{\theta+\theta_{0}}{2}\right) \mathrm{s} \gamma\right)
\end{array}\right\},\left\{\begin{array}{c}
-a(\mathrm{c} \alpha-\mathrm{c} \gamma) \mathrm{c} \alpha \mathrm{s}\left(\frac{\theta+\theta_{0}}{2}\right) \\
a(\mathrm{c} \alpha-\mathrm{c} \gamma) \operatorname{c} \alpha \mathrm{c}\left(\frac{\theta+\theta_{0}}{2}\right) \\
a(\mathrm{c} \alpha-\mathrm{c} \gamma) \mathrm{s} \alpha \mathrm{c}\left(\frac{\theta-\theta_{0}}{2}\right)
\end{array}\right\}\right),
$$

where "c" and "s" denote the cosine and sine functions.Huang $[3,4]$ has shown that this screw surface is a cylindroid. See Fig. 2 for several views of a cylindroid.
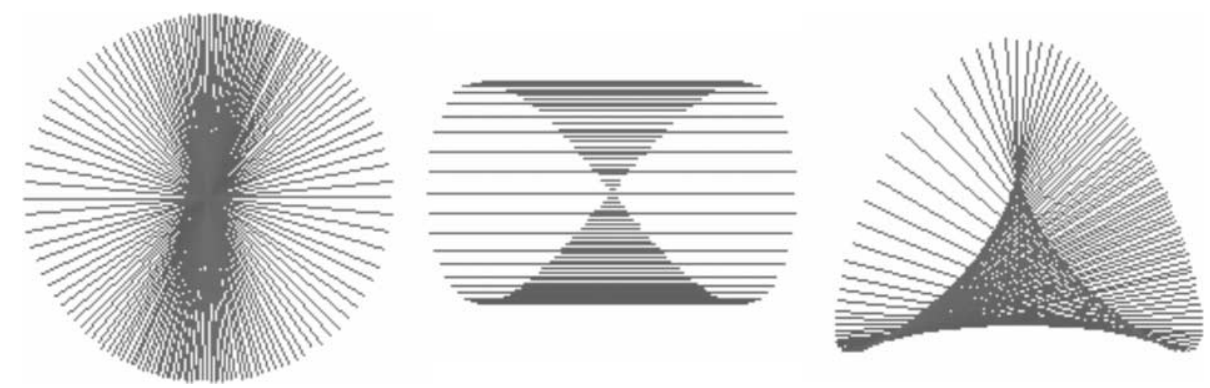

Fig. 2. The cylindroid as viewed first from the top along the central axis, then from the side and finally, from an angle. 
A change in the reference position,

$$
\left[D_{1 \theta}\right]=\left[D_{0 \theta}\right]\left[D_{10}\right],
$$

where $\left[D_{10}\right]=\left[D\left(\theta_{0}, \phi_{0}\right)\right]\left[D\left(\theta_{1}, \phi_{1}\right)\right]^{-1}$, is a right translation of the constraint manifold. It is important to note that this is not a rigid transformation of the screw surface. In what follows, we show how the cylindroid is affected by a change in reference position.

\section{The cylindroid}

A cylindroid is a ruled surface which is obtained as set of axes of the real linear combination of two screws. For example, let $\mathrm{U}$ and $\mathrm{V}$ be the dual vectors defining two screws, then the linear combination

$$
\mathrm{F}=a \mathrm{U}+b \mathrm{~V},
$$

where $a$ and $b$ are any real constants defines a one dimensional set of screws.

Let the axes of the screws $\mathrm{U}$ and $\mathrm{V}$ be $\mathrm{S}_{1}$ and $\mathrm{S}_{2}$, and their magnitudes and pitches be $M_{1}, P_{1}$ and $M_{2}, P_{2}$, respectively, so these screws can be written as

$$
\mathrm{U}=M_{1}\left(1, P_{1}\right) \mathrm{S}_{1}, \quad \mathrm{~V}=M_{2}\left(1, P_{2}\right) \mathrm{S}_{2} .
$$

Now determine the line $K$, which is the common normal to $S_{1}$ and $S_{2}$. Denote $S_{1}=I$ and compute $\mathrm{J}=\mathrm{K} \times \mathrm{I}$. Then, we can define $\mathrm{S}_{2}$ as

$$
\mathrm{S}_{2}=\cos \hat{\delta} \mathrm{I}+\sin \hat{\delta} \mathrm{J}
$$

where $\hat{\delta}=(\delta, d)$ is the dual angle from $\mathrm{S}_{1}$ to $\mathrm{S}_{2}$ measured around and along $\mathrm{K}$.

We can now write (20) as

$$
\begin{aligned}
& \mathrm{F}=a\left(1, P_{1}\right) \mathbf{I}+b\left(1, P_{2}\right)(\cos \hat{\delta} \mathbf{I}+\sin \hat{\delta} \mathrm{J}), \\
& \mathrm{F}=\left(a\left(1, P_{1}\right)+b\left(1, P_{2}\right) \cos \hat{\delta}\right) \mathbf{I}+b\left(1, P_{2}\right) \sin \hat{\delta} \mathrm{J},
\end{aligned}
$$

where we have absorbed the magnitudes $M_{1}$ and $M_{2}$ into the constants $a$ and $b$, respectively. This equation shows that the axes of all the screws $\mathrm{F}$ intersect $\mathrm{K}$ in a right angle. $\mathrm{K}$ is called the nodal line of the cylindroid.

Using the nodal line, the axes of screws of the cylindroid can be located by measuring the dual angle $\hat{\zeta}=(\zeta, z)$ relative to $\mathrm{I}=\mathrm{S}_{1}$ around the nodal line $\mathrm{K}$, that is

$$
\mathrm{F}=M(1, P)(\cos \hat{\zeta} \mathrm{I}+\sin \hat{\zeta} \mathrm{J}) .
$$

The parameter $z$ is called the offset and locates the intersection of the axis of each screw of the cylindroid with the nodal line K (Fig. 3).

We can equate (23) and (24) and eliminate $a$ and $b$ in order to derive a formula for the offset $z$ and pitch $P$ of each screw in the cylindroid,

$$
\left\{\begin{array}{c}
z(\zeta) \\
P(\zeta)
\end{array}\right\}=\left[\begin{array}{cc}
-\sin \zeta & \cos \zeta \\
\cos \zeta & \sin \zeta
\end{array}\right]\left[\begin{array}{cc}
P_{1} & \left(P_{2}-P_{1}\right) \cot \delta-d \\
0 & P_{2}+d \cot \delta
\end{array}\right]\left\{\begin{array}{c}
\cos \zeta \\
\sin \zeta
\end{array}\right\}
$$

The screws with the maximum and minimum values of the pitch $P$ are the principal axes of the cylindroid. Set the derivative of the above expression to zero, to obtain 

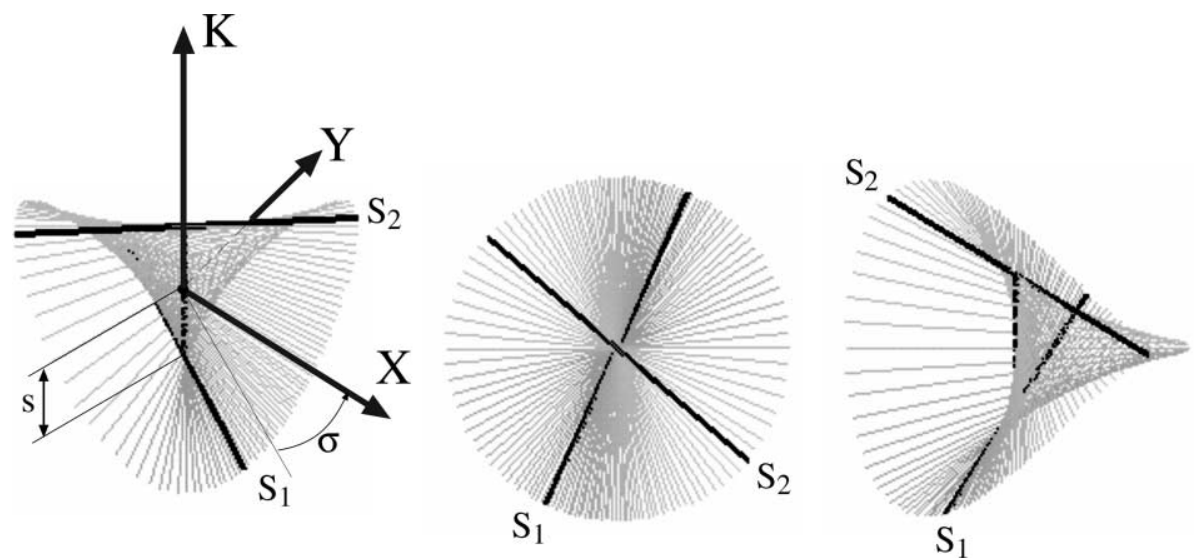

Fig. 3. The principal axes as located relative to the screw axes $S_{1}$ and $S_{2}$.

$$
\frac{\mathrm{d}}{\mathrm{d} \zeta}\left\{\begin{array}{c}
z \\
P
\end{array}\right\}=\left[\begin{array}{cc}
-\left(P_{2}-P_{1}\right) \cot \delta+d & \left(P_{2}-P_{1}\right)+d \cot \delta \\
\left(P_{2}-P_{1}\right)+d \cot \delta & \left(P_{2}-P_{1}\right) \cot \delta-d
\end{array}\right]\left\{\begin{array}{c}
\sin 2 \zeta \\
\cos 2 \zeta
\end{array}\right\}=\left\{\begin{array}{l}
0 \\
0
\end{array}\right\} .
$$

The angles $\zeta=\sigma$ with extreme values of $P$ are given by

$$
\tan 2 \sigma=\frac{-\left(P_{2}-P_{1}\right) \cot \delta+d}{\left(P_{2}-P_{1}\right)+d \cot \delta}
$$

which yields two angles separated by $\pi / 2$. This defines the directions of principal axes $X$ and $Y$ of the cylindroid. The offset $s$ to the principal axes is given by substituting $\sigma$ into Eq. (25) to obtain

$$
s=\frac{1}{2}\left(d-\left(P_{2}-P_{1}\right) \frac{\cos \delta}{\sin \delta}\right) \text {. }
$$

Thus, the principal axes are located by the dual angle $\hat{\sigma}=(\sigma, s)$, as

$$
X=\cos \hat{\sigma} l+\sin \hat{\sigma} J, \quad Y=-\sin \hat{\sigma} l+\cos \hat{\sigma} J .
$$

The height of the cylindroid is the distance along $\mathrm{K}$ between minimum and maximum offsets. This is calculated from from Eq. (26) to be

$$
L_{\mathrm{c}}=\frac{\sqrt{\left(P_{2}-P_{1}\right)^{2}+d^{2}}}{\sin \delta} .
$$

If the principal screws are used to define the cylindroid, then $\hat{\delta}=(\delta, d)=(\pi / 2,0)$, and the height is given by [5]:

$$
L_{\mathrm{c}}=\left|P_{Y}-P_{X}\right| \text {. }
$$

\section{The Bennett tetrahedron}

The reference configuration of the Bennett linkage defines locations of the points $\mathbf{B}, \mathbf{P}, \mathbf{C}$, and $\mathbf{Q}$ that form a tetrahedron. This tetrahedron is directly related to the cylindroid generated by the relative displacement screw for this reference configuration. 
Introduce the line $\mathrm{L}_{\mathrm{CB}}$ that forms the edge of the tetrahedron defined by the segment $\mathbf{C B}$. Similarly, let $L_{P Q}$ be the line defined by $\mathbf{P Q}$. The dual vectors for these lines are easily calculated to be

$$
\mathrm{L}_{\mathrm{CB}}=(\mathbf{B}-\mathbf{C}, \mathbf{B} \times(\mathbf{B}-\mathbf{C})) \quad \text { and } \quad \mathrm{L}_{\mathrm{PQ}}=(\mathbf{Q}-\mathbf{P}, \mathbf{Q} \times(\mathbf{Q}-\mathbf{P})) .
$$

Using the expressions of the points $\mathbf{B}, \mathbf{Q}, \mathbf{P}$ and $\mathbf{C}$ in the reference frame $F$, we can compute

$$
\begin{aligned}
\mathrm{L}_{\mathrm{CB}} & =\left(\left\{\begin{array}{c}
\sin \alpha \cos \gamma \cos \theta_{0}-\cos \alpha \sin \gamma \\
\sin \alpha \cos \gamma \sin \theta_{0} \\
\sin \alpha \sin \gamma \sin \theta_{0}
\end{array}\right\},\left\{\begin{array}{l}
0 \\
0 \\
0
\end{array}\right\}\right) \text { and } \mathrm{L}_{\mathrm{PQ}} \\
& =\left(\left\{\begin{array}{c}
\sin \gamma-\sin \alpha \cos \theta_{0} \\
-\sin \alpha \sin \theta_{0} \\
0
\end{array}\right\},\left\{\begin{array}{c}
0 \\
0 \\
-g \sin \alpha \sin \theta_{0}
\end{array}\right\}\right) .
\end{aligned}
$$

We now show that the this tetrahedron is symmetric relative to the line joining the midpoints of the edges $\mathbf{C B}$ and $\mathbf{P Q}$. In particular, this line is the common normal to the two lines $\mathrm{L}_{\mathrm{CB}}$ and $\mathrm{L}_{\mathrm{PQ}}$. Define the midpoints $\mathbf{V}_{1}$ and $\mathbf{V}_{2}$, such that

$$
\mathbf{V}_{1}=\frac{\mathbf{B}+\mathbf{C}}{2}=\left\{\begin{array}{c}
\frac{1}{2}\left(g+a \cos \psi_{0}\right) \\
\frac{1}{2}\left(a \cos \gamma \sin \psi_{0}\right) \\
\frac{1}{2}\left(a \sin \gamma \sin \psi_{0}\right) \\
1
\end{array}\right\} \quad \text { and } \quad \mathbf{V}_{2}=\frac{\mathbf{P}+\mathbf{Q}}{2}=\left\{\begin{array}{c}
\frac{1}{2}\left(g+a \cos \theta_{0}\right) \\
\frac{1}{2}\left(a \sin \theta_{0}\right) \\
0 \\
1
\end{array}\right\} \text {. }
$$

The vector $\mathbf{T}=\mathbf{V}_{2}-\mathbf{V}_{1}$ is perpendicular to the lines $\mathrm{L}_{\mathrm{CB}}$ and $\mathrm{L}_{\mathrm{PQ}}$, if it is perpendicular to the direction components, $\mathbf{L}_{1}$ and $\mathbf{L}_{2}$, respectively. Computing the $\operatorname{dot} \operatorname{product} \mathbf{T} \cdot \mathbf{L}_{1}$, we find that

$$
\left(\mathbf{V}_{2}-\mathbf{V}_{1}\right) \cdot \mathbf{L}_{1}=(a / 2)((g-a \cos \theta) \cos \psi-(a \cos \gamma \sin \theta) \sin \psi+(a+g \cos \theta))=0 .
$$

A similar calculation yields $\mathbf{T} \cdot \mathbf{L}_{2}=0$. The line along $\mathbf{T}$ is the axis of the Bennett tetrahedron.

The dual cross product $\mathrm{T}=\mathrm{L}_{\mathrm{CB}} \times \mathrm{L}_{\mathrm{PQ}}$ defines a screw along the direction $\mathbf{T}$,

$$
\mathrm{T}=\left(\left\{\begin{array}{c}
\sin \alpha \sin \theta_{0} \\
\sin \gamma-\sin \alpha \cos \theta_{0} \\
\cos \alpha-\cos \gamma
\end{array}\right\},\left\{\begin{array}{c}
-g \cot \gamma \sin \alpha \sin \theta_{0} \\
g\left(\cot \gamma \sin \alpha \cos \theta_{0}-\cos \alpha\right) \\
0
\end{array}\right\}\right) \text {. }
$$

Compute the dual dot product

$$
\mathbf{F}^{\prime}\left(\theta, \theta_{0}\right) \cdot \mathbf{T}=\left(\mathbf{F}, \mathbf{F}^{0}\right) \cdot\left(\mathbf{T}, \mathbf{T}^{0}\right)=\left(\mathbf{F} \cdot \mathbf{T}, \mathbf{F}^{0} \cdot \mathbf{T}+\mathbf{F} \cdot \mathbf{T}^{0}\right)=(0,0),
$$

which shows that the axis of $\mathrm{T}$ is the common normal to each of the axes of the screws $\mathrm{F}^{\prime}$. This provides the primary result of our paper, the axis of the Bennett tetrahedron is the nodal line of the cylindroid of relative screw axes.

\section{The reference pyramid}

The axis of the Bennett tetrahedron is known to be the line of symmetry for the movement of the Bennett linkage, (see $[8,13]$ ). We denote the axis of the Bennett tetrahedron by $\mathrm{K}$, as it 
coincides with the nodal line of the cylindroid. We now construct a rectangular pyramid with $\mathrm{K}$ as its axis, that contains this tetrahedron. This pyramid provides a convenient way to find the principal axes of the cylindroid (Fig. 4).

Consider the rectangle in a plane perpendicular to $K$ that has vertices $\mathbf{B}$ and $\mathbf{C}$ on its diagonal. Similarly, consider the second rectangle perpendicular to $K$, that has vertices $\mathbf{P}$ and $\mathbf{Q}$ on its diagonal. These two rectangles are cross-sections of a rectangular pyramid. The four vertices of these two cross-sections are labeled $\mathbf{A}_{i}$ and $\mathbf{B}_{i}, i=1,2,3,4$ counter-clockwise around the axis $\mathrm{K}$. The vertices

$$
\mathbf{A}_{1}=\mathbf{B}, \quad \mathbf{B}_{2}=\mathbf{Q}, \quad \mathbf{A}_{3}=\mathbf{C}, \quad \mathbf{B}_{4}=\mathbf{P}
$$

define the Bennett tetrahedron.

We now assume that the length $m=|\mathbf{Q}-\mathbf{P}|$ is less than the length $n=|\mathbf{C}-\mathbf{B}|$, which means the cross-section $\mathbf{A}_{i}$ can be viewed as the base of the reference pyramid. Introduce the coordinate frame $M^{\prime}$ with its $z$-axis along $\mathrm{K}$, and its $x$-axis directed along $\mathbf{A}_{1}-\mathbf{A}_{2}$, which means its $y$-axis is directed along $\mathbf{A}_{1}-\mathbf{A}_{4}$. This allows us to define the coordinates of $\mathbf{A}_{i}$ as

$$
\mathbf{A}_{1}=\left\{\begin{array}{l}
r \\
s \\
0 \\
1
\end{array}\right\}, \quad \mathbf{A}_{2}=\left\{\begin{array}{c}
-r \\
s \\
0 \\
1
\end{array}\right\}, \quad \mathbf{A}_{3}=\left\{\begin{array}{c}
-r \\
-s \\
0 \\
1
\end{array}\right\}, \quad \mathbf{A}_{4}=\left\{\begin{array}{c}
r \\
-s \\
0 \\
1
\end{array}\right\} .
$$

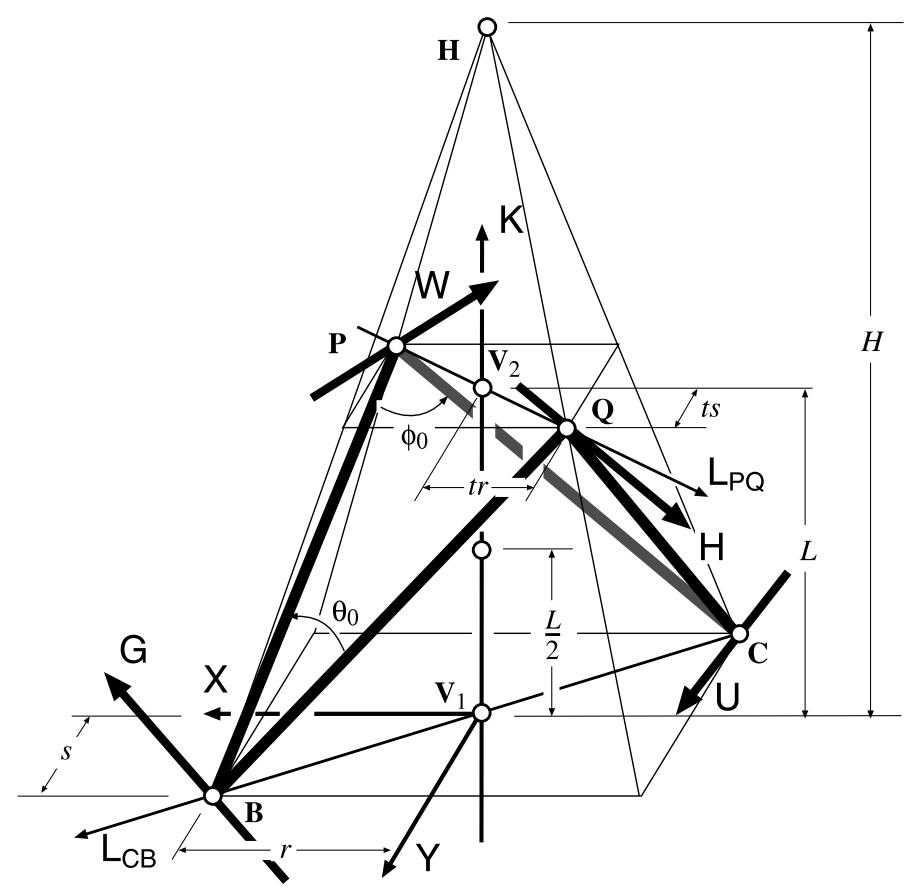

Fig. 4. The pyramid associated with the Bennett Linkage GWUH. 
The coordinates of the points $\mathbf{A}_{i}$ and $\mathbf{B}_{i}$ are related by the fact that they lie parallel cross-sections of the pyramid. Let $\mathbf{H}=(0,0, H, 1)^{\mathrm{T}}$ define the apex of the pyramid measured from the first crosssection along $\mathrm{K}$, then we have

$$
\mathbf{B}_{i}=(1-t) \mathbf{A}_{i}+t \mathbf{H}, \quad i=1, \ldots, 4 .
$$

The parameter $t$ ranges between 0 and 1 . The value of $t$ that locates the second cross-section is determined by computing the ratio of the lengths $m=|\mathbf{Q}-\mathbf{P}|$ and $n=|\mathbf{C}-\mathbf{B}|$, that is

$$
t=1-\frac{m}{n}
$$

The lengths $m$ and $n$ are found using the cosine law, that is

$$
m=\sqrt{a^{2}+g^{2}-2 a g \cos \theta_{0}}, \quad n=\sqrt{a^{2}+g^{2}+2 a g \cos \phi_{0}} .
$$

The distance $L$ between the two cross-sections and the height $H$ of the pyramid are related by the formula

$$
L=t H
$$

The value of $L$ is obtained by evaluating the magnitude of the vector joining the midpoints $\mathbf{V}_{1}$ and $\mathbf{V}_{2}$, which, after some algebra, is given by

$$
L=\left|\mathbf{V}_{2}-\mathbf{V}_{1}\right|=\sqrt{\frac{1}{2} a g\left(\cos \theta_{0}-\cos \phi_{0}\right)} .
$$

In order to complete the specification of this pyramid, we determine the lengths $r$ and $s$ that locate the points $\mathbf{A}_{i}$. This is done using the identities $a^{2}=\left(\mathbf{B}_{4}-\mathbf{A}_{1}\right) \cdot\left(\mathbf{B}_{4}-\mathbf{A}_{1}\right)$ and $g^{2}=$ $\left(\mathbf{B}_{2}-\mathbf{A}_{1}\right) \cdot\left(\mathbf{B}_{2}-\mathbf{A}_{1}\right)$, which yield the matrix equation

$$
\left\{\begin{array}{cc}
t^{2} & (t-2)^{2} \\
(t-2)^{2} & t^{2}
\end{array}\right\}\left\{\begin{array}{l}
r^{2} \\
s^{2}
\end{array}\right\}=\left\{\begin{array}{l}
a^{2}-(t H)^{2} \\
g^{2}-(t H)^{2}
\end{array}\right\} .
$$

This is easily solved to determine the values for $r$ and $s$.

The parameters $r, s, t$ and $L$ completely define the reference pyramid. They are obtained from the reference configuration of the Bennett linkage as defined by the specified input angle $\theta=\theta_{0}$ (Fig. 5).

The coordinate transformation $[D]=[A, \mathbf{d}]$ from the standard frame $F$ to the frame $M^{\prime}$ located on the base of the pyramid in Fig. 4 can be obtained from the matrix expression

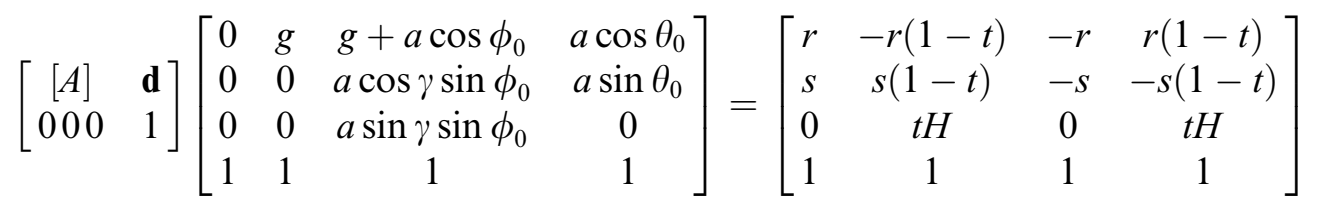

Given the coordinates of these points in both frames, we can multiply on the right by the inverse of the matrix defined by points in $F$ to compute the transformation $[D]$. 


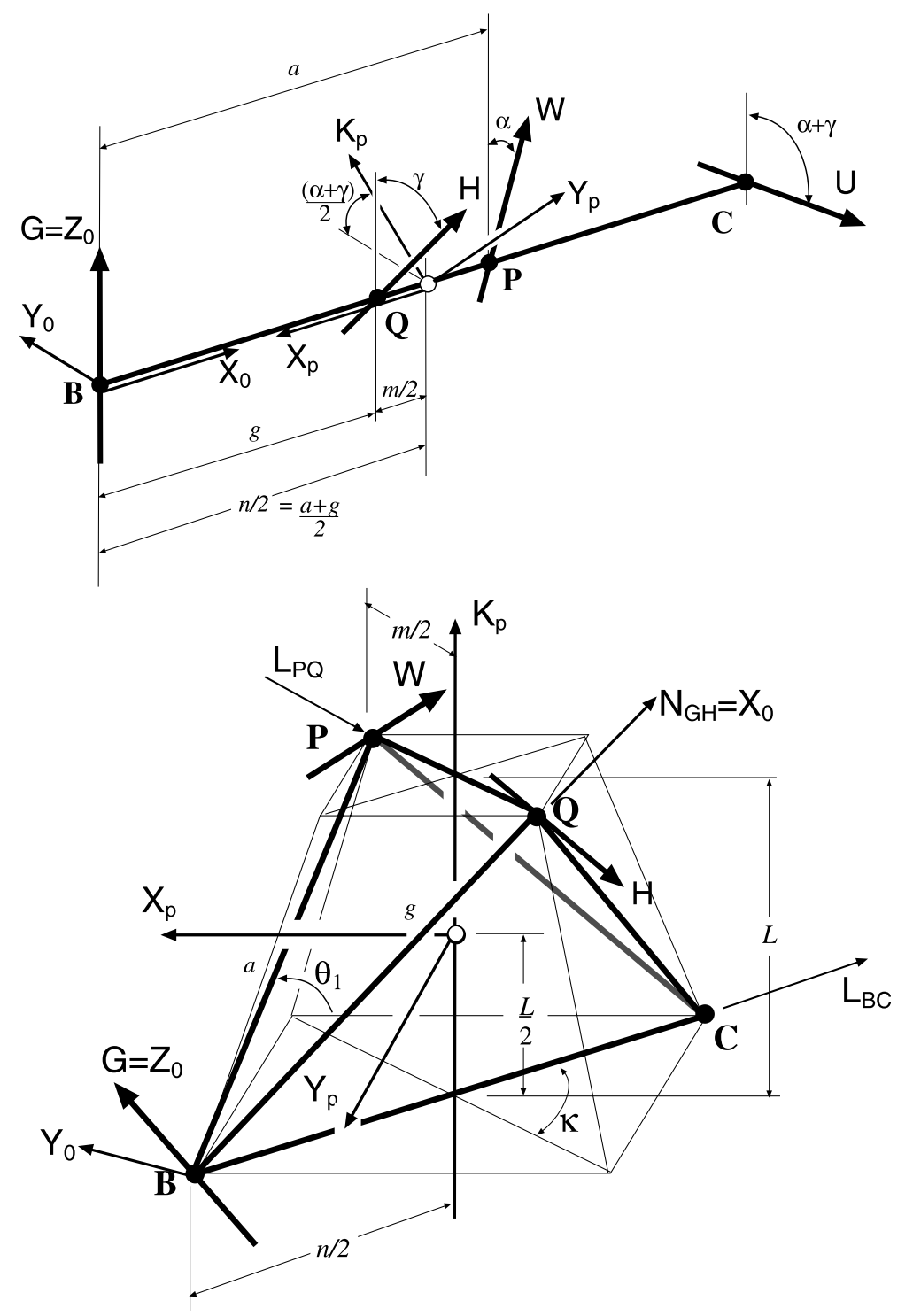

Fig. 5. The Bennett linkage with initial angle $\theta=0$ and $\theta=\theta_{1}$.

\section{The cylindroid and the reference position}

We illustrate the relation between the cylindroid and the reference position with examples. The nodal line of the cylindroid is along the K-axis of the tetrahedron defined by the reference configuration of the linkage. A change of reference configuration corresponds to a right translation of the workspace and therefore of the cylindroid. This is not a rigid transformation of the screws of the cylindroid. 


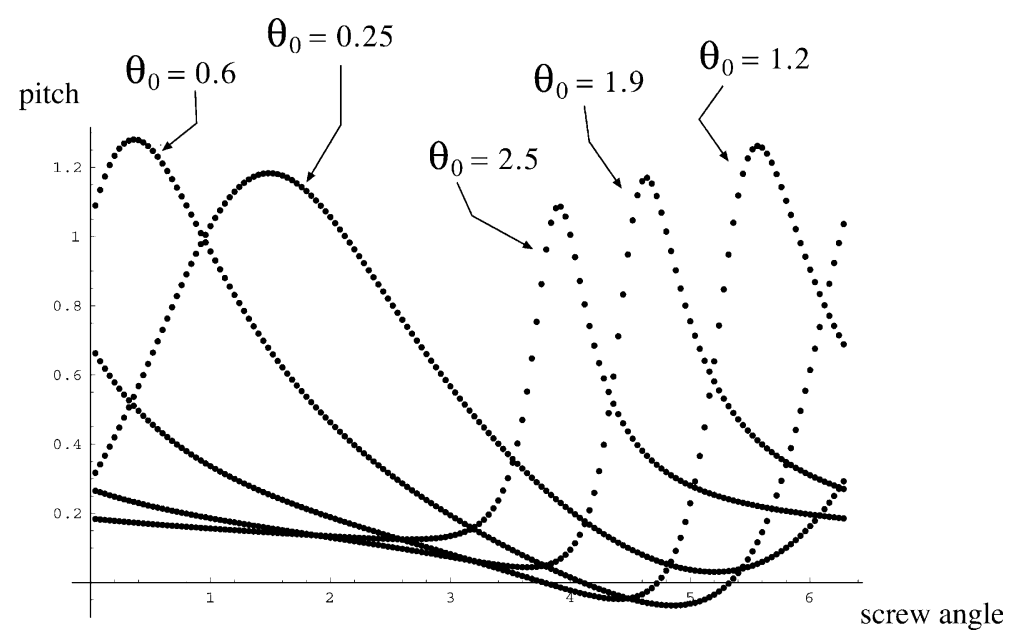

Fig. 6. The distribution of screw pitches in cylindroids associated with various reference configurations of Linkage 1.

Consider the Bennett linkages with the two sets of dimensions:

(1) Linkage 1: $a=1.1, g=1.3, \alpha=0.8 \mathrm{rad}$;

(2) Linkage 2: $a=0.76, g=2.22, \alpha=0.27 \mathrm{rad}$.

The first has nearly equal values for the dimensions $a$ and $g$, which, in our experience, yields sharper differences between cylindroids of various reference positions. The second example has a larger difference between the values of $a$ and $g$, which yields cylindroids that are generally similar.

Fig. 6 shows the distribution of pitches in the cylindroid for various choices of the reference angle $\theta_{0}$ for Linkage 1 . Fig. 7 shows the distribution of pitches for Linkage 2. The variation in location and shape of these profiles is the result of the right translation of the cylindroid.

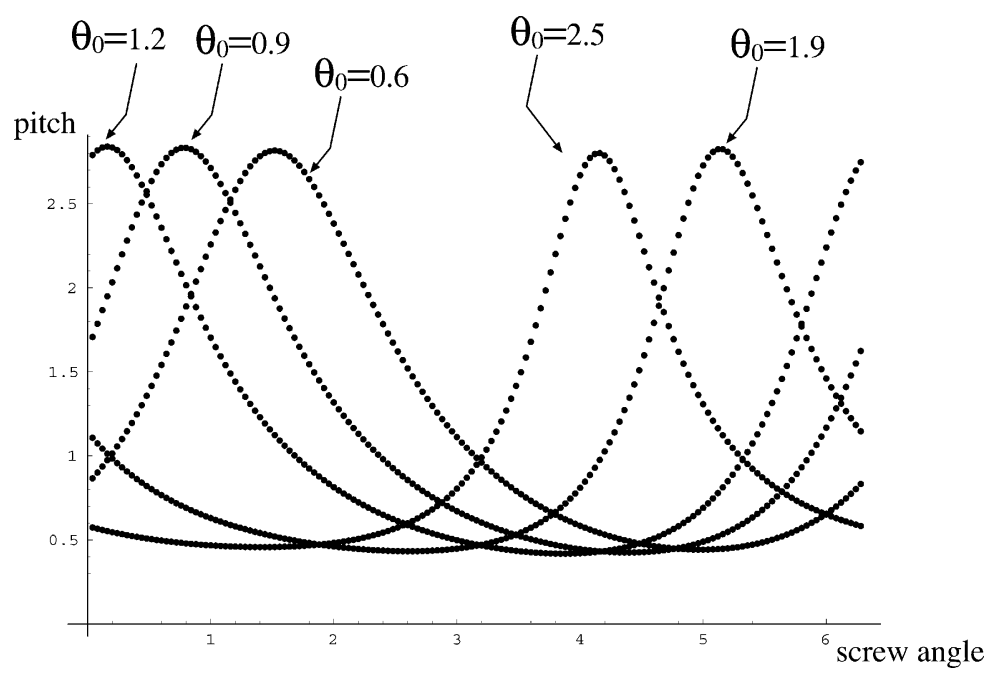

Fig. 7. The distribution of screw pitches for Linkage 2. 

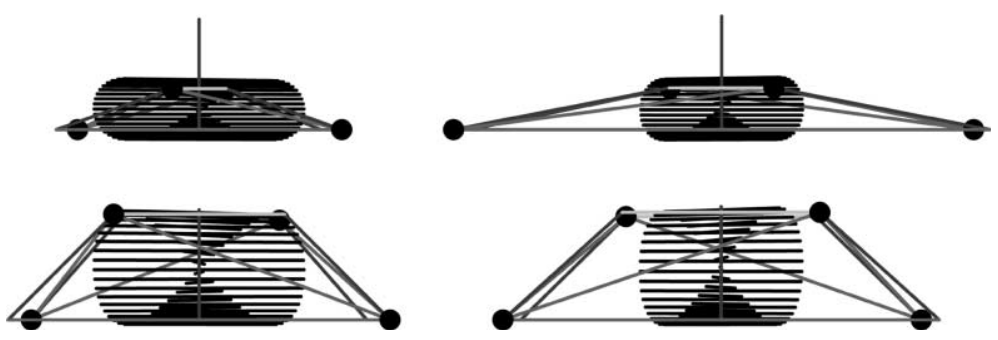

Fig. 8. Linkage 1: front and right side views of the cylindroid and Bennett tetrahedron in two reference configurations.
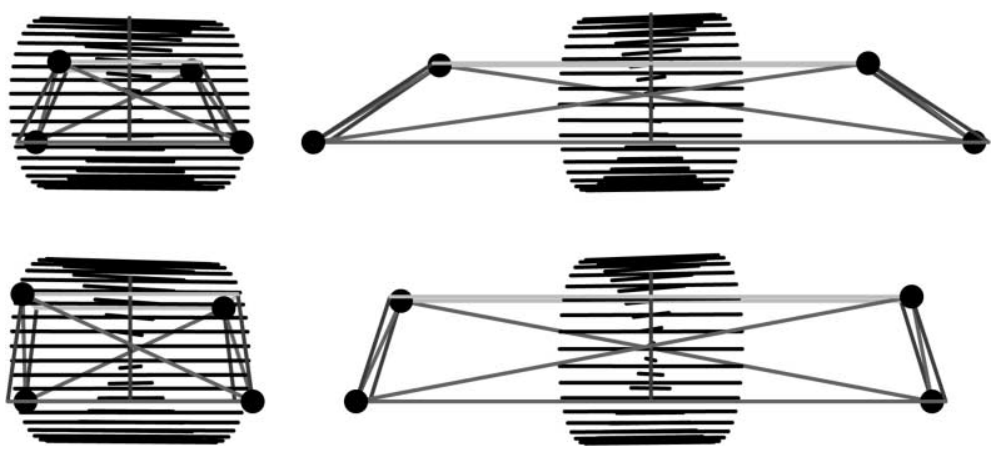

Fig. 9. Linkage 2: front and right side views of the cylindroid and Bennett tetrahedron in two reference configurations.

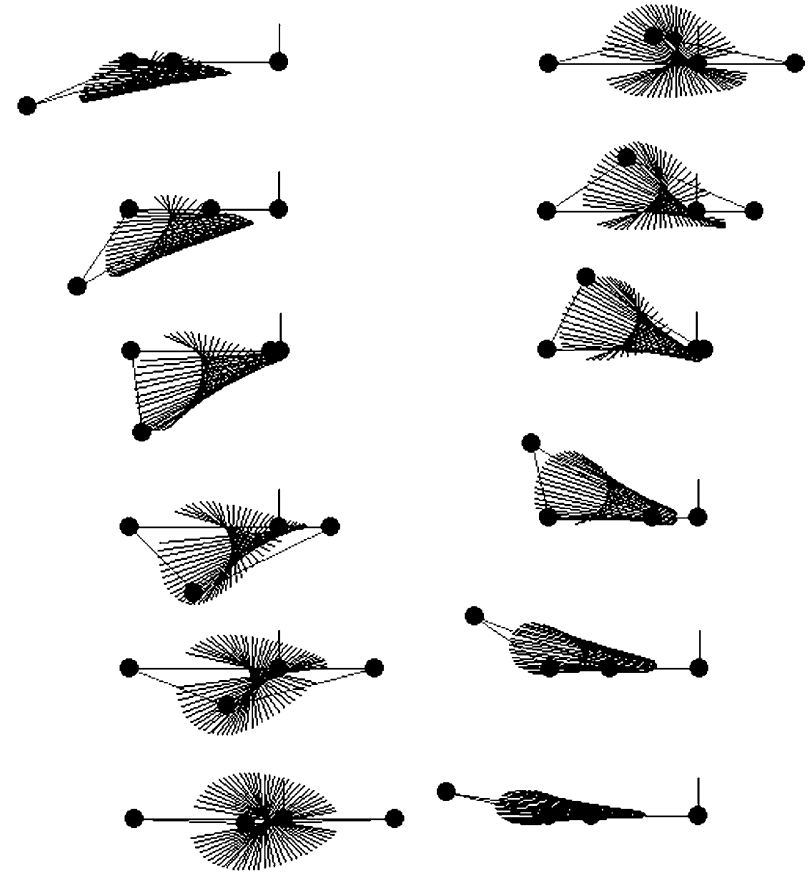

Fig. 10. The change in position and orientation of the cylindroid for Linkage 1 for twelve different reference configurations. 
The relationship between the height of the cylindroid and the Bennett tetrahedron for two reference configurations is shown in Fig. 8 for Linkage 1, and Fig. 9 for Linkage 2. These figures show front and right side views of the cylindroid and Bennett tetrahedron. They were generated using Mathematica routines which are available from the authors upon request.

In example 1, we see that the height of the cylindroid varies considerably between the two reference configurations. This is not the case for example 2.

In Fig. 10 we can see the changes in the cylindroid as different configurations of the Bennett linkage are used as the reference position.

The equations relating the cylindroid and a general reference configuration require further study. However, the cylindroid associated with the configuration defined by $\theta_{0}=0$ has a simple form and is examined in the next section.

\section{The folded configuration}

The screw $\mathrm{T}$, which defines the nodal line of the cylindroid, takes a simple form if the initial configuration of the Bennett linkage is $\theta_{0}=0$. In this configuration the the linkage folds to form a straight line (Fig. 5).

Introduce the angles $\xi=(\alpha+\gamma) / 2$ and $\eta=(\alpha-\gamma) / 2$, substitute $\alpha=\xi+\eta$ and $\gamma=\xi-\eta$ into (36), and drop the common factor to obtain

$$
\mathrm{T}=\left(\left\{\begin{array}{c}
0 \\
\cos \xi \\
\sin \xi
\end{array}\right\},\left\{\begin{array}{c}
0 \\
g \frac{\cos \eta}{\sin (\eta-\xi)} \\
0
\end{array}\right\}\right)
$$

It is easy to see that the axis of $\mathrm{T}$ is directed along a line that bisects the axes of $\mathrm{G}$ and $\mathrm{U}$ parallel to the $y z$ plane. We determine a point $\mathbf{r}$ on the axis of $\mathrm{T}=\left(\mathbf{T}, \mathbf{T}^{0}\right)$ using the formula

$$
\mathbf{r}=\frac{\mathbf{T} \cdot \mathbf{T}^{0}}{\mathbf{T} \cdot \mathbf{T}}=\left\{\begin{array}{c}
\frac{a+g}{2} \\
0 \\
0
\end{array}\right\}
$$

In this calculation, we have used the fact that $a=g \sin \alpha / \sin \gamma$. Thus, the axis of T intersects the $x$ axis midway along the length of the folded linkage.

We now introduce the coordinate frame $M$ with its $y$-axis aligned the $x$-axis of $F$, its origin at $\mathbf{r}$ and its $z$-axis along T. Coordinates in the frame $F$ are transformed to $M$ by the $6 \times 6$ matrix,

$$
[\widehat{T}]=\left[\begin{array}{cccccc}
0 & \sin \xi & -\cos \xi & 0 & 0 & 0 \\
-1 & 0 & 0 & 0 & 0 & 0 \\
0 & \cos \xi & \sin \xi & 0 & 0 & 0 \\
0 & 0 & 0 & 0 & \sin \xi & -\cos \xi \\
0 & 0 & \frac{g+a}{2} & -1 & 0 & 0 \\
0 & -\frac{g+a}{2} & 0 & 0 & \sin \xi & -\cos \xi
\end{array}\right]
$$


Applying this transformation to the screws $\mathrm{F}^{\prime}$ in the cylindroid (18), we obtain

$$
\mathrm{F}^{\prime}=\left(\left\{\begin{array}{c}
\cos \frac{\theta}{2} \frac{1}{\sin \xi} \\
\sin \frac{\theta}{2} \\
0
\end{array}\right\},\left\{\begin{array}{c}
g \cos \frac{\theta}{2} \frac{\sin \eta}{\sin (\eta-\xi)} \\
-g \sin \frac{\theta}{2} \frac{\cos (\xi+\eta)}{\sin (\eta-\xi)} \\
0
\end{array}\right\}\right)
$$

The zero values in the $z$ components of the two vectors defining $\mathrm{F}^{\prime}$ show that the $z$-axis of $M$ is the nodal line of this cylindroid.

In order to compute the principal screws, we choose two arbitrary screws of this cylindroid and apply the formulas of the earlier section. For this purpose the screws $\mathrm{U}=\mathrm{F}^{\prime}(0)$ and $\mathrm{V}=\mathrm{F}^{\prime}(\pi)$ are convenient choices. We have

$$
\mathrm{U}=\left(\left\{\begin{array}{c}
\frac{1}{\sin \xi} \\
0 \\
0
\end{array}\right\},\left\{\begin{array}{c}
g \frac{\sin \eta}{\sin (\eta-\xi)} \\
0 \\
0
\end{array}\right\}\right), \quad \mathrm{V}=\left(\left\{\begin{array}{l}
0 \\
1 \\
0
\end{array}\right\},\left\{\begin{array}{c}
0 \\
-g \frac{\cos (\xi+\eta)}{\sin (\eta-\xi)} \\
0
\end{array}\right\}\right) .
$$

The axes of these screws, denoted $\mathrm{X}$ and $\mathrm{Y}$, are easily seen to be the $x$ and $y$ axes of $M$. Therefore, the angle between them is $\delta=\pi / 2$ and the normal distance is $d=0$. Eqs. (27) and (28) yield $\sigma=0$ and $s=0$ which means that $\mathrm{X}$ and $\mathrm{Y}$ are, in fact, the principal axes of the cylindroid $\mathrm{F}^{\prime}$.

We can now calculate the pitch $P(\theta)$ of each of the screws in the cylindroid to be

$$
P(\theta)=\left(\frac{g \sin \xi}{\sin (\eta-\xi)}\right)\left(\frac{\cos \left(\frac{\theta}{2}\right)^{2} \sin \eta-\sin \left(\frac{\theta}{2}\right)^{2} \sin \xi \cos (\eta+\xi)}{\cos \left(\frac{\theta}{2}\right)^{2}+\sin \left(\frac{\theta}{2}\right)^{2} \sin \xi^{2}}\right) .
$$

The $z$ coordinate of their intersection with the axis of $\mathrm{T}$ is given by

$$
z(\theta)=\frac{-g \sin \xi \cos \xi \sin \theta}{2 \sin (\eta-\xi)\left(\cos \left(\frac{\theta}{2}\right)^{2}+\sin \left(\frac{\theta}{2}\right)^{2} \sin \xi^{2}\right)} .
$$

This provides a complete characterization of the cylindroid associated with Bennett's linkage when displacements of the coupler are measured relative to its folded configuration.

\section{Conclusion}

This paper examines the screw axes of the constraint manifold of the coupler of Bennett's linkage. These screws form a cylindroid, which is a linear combination of two screws.

The choice of reference position of the linkage results in a right translation of the constraint manifold, which is not a coordinate transformation of relative displacement screws. Examples demonstrate the relationship between the reference position and the properties of this cylindroid. The folded configuration of the linkage is shown to provide a convenient frame in which to study this cylindroid.

These results are important to understanding the geometry of the workspace of the RR chain, which is the simplest robot manipulator. For a given RR chain, $\hat{\alpha}=(\alpha, a)$, we can select $g$ and compute $\gamma$ to obtain a parameterized set of Bennett motions contained in this workspace. 


\section{References}

[1] G.T. Bennett, A new mechanism, Engineering 76 (1903) 777-778.

[2] J.M. McCarthy, Introduction to Theoretical Kinematics, The MIT Press, Cambridge, MA, 1990.

[3] C. Huang, The cylindroid associated with finite motions of the Bennett mechanism, Proceedings of the ASME Design Engineering Technical Conferences, Irvine, CA, 1996.

[4] C. Huang, On the finite screw system of the third order associated with a revolute-revolute chain, Journal of Mechanical Design 116 (1994) 875-883.

[5] K.H. Hunt, Kinematic Geometry of Mechanisms, Clarendon Press, Oxford, 1978.

[6] O. Bottema, B. Roth, Theoretical Kinematics, North-Holland, Amsterdam, 1979, reprinted by Dover Publications, 1990.

[7] I.A. Parkin, Finding the principal axes of screw systems, Proceedings of the ASME Design Engineering Technical Conferences, Sacramento, CA, 1997.

[8] H.C. Yu, The Bennett linkage, its associated tetrahedron and the hyperboloid of its axes, Mechanism and Machine Theory 16 (1981) 105-114.

[9] A. Perez, J.M. McCarthy, Dimensional synthesis of Bennett linkages, Proceedings of the ASME Design Engineering Technical Conferences, Baltimore, MD, 10-13 September 2000.

[10] K. Hao, Dual number method, rank of a screw system and generation of lie sub-algebras, Mechanism and Machine Theory 33 (7) (1998) 1063-1084.

[11] J.M. McCarthy, Geometric Design of Linkages, Springer-Verlag, Berlin, 2000.

[12] F. Dimentberg, The screw calculus and its applications in mechanics, US Department of Commerce, Translation No. AD680993, 1965.

[13] J.E. Baker, On the motion geometry of the Bennett linkage, in: Proceedings of the 8th International Conference on Engineering Computer Graphics and Descriptive Geometry, Austin, Texas, USA, vol. 2, 1998, pp. 433-437. 